\title{
Application of Intelligent Sensing in Power Test System
}

\author{
Tianpeng Zhang", Bingwei Wang \\ Mechanical Engineering School,Yellow River conservancy Technical Institute, Kaifeng, Henan, China \\ *Corresponding Author.
}

\begin{abstract}
Smart grid is the development trend of the power industry under the new situation. As an important part of smart grid, smart power consumption directly faces the society and power users, and plays a very important role in the construction of smart grid. With the development of intelligent power construction, the traditional power service can not meet the needs of users to participate in demand side management and interact with the power grid in time. Therefore, the construction of a new intelligent sensing power measurement system to improve the quality and level of intelligent power service has become the focus of current power industry research. Firstly, according to the requirements analysis and design principles of the intelligent sensing power detection system, this paper proposes the function analysis and three-tier architecture design of the intelligent sensing power detection system. The intelligent sensing power detection system is logically divided into business application layer, network communication layer and sensing device layer. Externally, it realizes data integration and interaction with power consumption information collection system, marketing business management system and other intelligent power consumption information systems. Internally, it realizes interconnection with user side perception interactive terminal and perception unit, and realizes data acquisition, information transmission and control functions of power consumption terminal equipment. This paper presents a method of applying intelligent sensing technology to data editing environment of power simulation software, and realizes online error detection of simulation original data, automatic completion of keywords, automatic prompt of data card and intelligent help of data domain. The power simulation data editing software with intelligent sensing function developed in this paper is suitable for primary and advanced users, which can improve work efficiency and reduce error rate. The configurable rule management method applied in the implementation of intelligent sensing technology extends the application of data editor to the developers of models and algorithms, and provides a simple way for the timely upgrade and release of new models and algorithms.
\end{abstract}

Keywords: Smart grid, smart sensing, configurable rules, power detection.

\section{Introduction}

Power system simulation plays an important role in power research, planning, design, operation, test and training. Generally, power system simulation software mainly includes electromechanical simulation, electromagnetic transient simulation and hybrid simulation. Foreign electromechanical simulation software includes PSS / E [1-2] of PTI, ETMSP of ERPI, NETOMAC of Siemens and SIMPOW [3] of ABB in Switzerland; domestic electric power Research Institute of China.

PSD (including Chinese version of BPA) and PSASP integrated program is used in this paper. Foreign electromagnetic transient simulation software includes EMTP of Bonneville Power Administration (BPA), PSCAD / EMTDC of Manitoba DC research center in Canada [3], NETOMAC of Siemens [4], PSD and PSASP of China Electric Power Research Institute in China [5-6], DDRTS [7] jointly developed by Yintu company and Tsinghua University. In terms of hybrid simulation, China Electric Power Research Institute and Tsinghua University are carrying out relevant research and development [8].

With the rapid development of power industry, simulation software has made great progress in model structure (such as load model, facts element model, AC / DC hybrid model, excitation characteristics, governor model,

ISSN: 0010-8189

(C) CONVERTER 2020 
etc.), model parameters [9], simulation algorithm and method, simulation speed, scale, time and comparative study [10]. However, there are few researches on how to reduce the construction time of simulation raw data and how to reduce data entry errors.

The first step of simulation is to build the original simulation data, such as model parameters, network topology parameters, simulation control parameters (such as algorithm selection, calculation accuracy step, calculation simulation type). These data need to be adjusted repeatedly according to the simulation requirements, which is quite time-consuming work. According to the form of construction and storage, the way to create the original simulation data can be divided into three types: 1) text type, the simulation data is directly constructed by writing data card and control card, and the data is in ASCII Format storage, in the editor to write and modify, change directly and quickly, data independence, easy to transfer and share, but requires users to be very familiar with the definition and limitations of data card and control card, higher requirements for users; 2) database type, is a form of input through the form of tables and dialog box simulation of the original data, co-exist in the database, this way is easy to use, but is limited Limited to the requirements of database format, the flexibility and sharing are not strong; 3) graphic type, which represents the physical components and connection relations through symbols and attributes, and uses session box to set attributes and parameters, is easy to use and friendly to display, but the data is generally stored in binary format, so it is difficult to share and interact due to the poor intuitiveness of attribute search and comparison.

PSD software package is widely used in universities, scientific research institutions, planning and operation departments. The simulation data construction environment based on text mode is favored by skilled users. However, due to the strict definition of data format, it is difficult for beginners to use it. In view of this situation, this paper proposes to use the intelligent sensing technology to simulate the data editing environment, which can improve the ease of use and data construction efficiency of the data editor while retaining the advantages of text input. Intelligent sensing technology is a way to provide users with timely services through context aware computing, that is, by capturing the state information of the physical environment of users, inferring the possible needs of users according to their historical behavior, and providing corresponding services in a timely manner.

This paper will discuss the implementation methods and examples of intelligent sensing technology based on the definition of PSD simulation editing software model card and control card, summarize the characteristics of intelligent sensing technology editing environment, and look forward to the future application of intelligent sensing technology in power simulation software.

\section{Implementation method and main functions of intelligent perception of editing environment}

Intelligent perception realizes operation target positioning through location perception. The positioning target is used as the trigger signal of intelligent agent. Through intelligent agent calculation, judgment and screening, timely information is released to realize dynamic interaction with users, and online help, customized task automatic service and content online error detection are provided for users.

In the data editing environment, perception has a wide range of applications, such as editing line number, ruler, label, annotation, right-click menu, status bar prompt, mouse shape, keyword coloring, which are all dynamic feedback after location perception. Primary applications can't reflect the intelligent function of perception well, so we must design intelligent service agent in editing environment, build special rules and application experience configuration library to provide timely, convenient and needed intelligent services for users.

Intelligent perception of data editing environment is mainly composed of configuration library, location aware and intelligent agent. The configuration library is designed according to the internal requirements of the simulation software, including format specification, calculation or control keywords and data card format definition. Take PSD power flow analysis as an example], symbol ()$, / \ldots \backslash,>_{\ldots}<$ indicates the control statement (middle Powerflow, caseid, nextcase, end, stop, etc. are used to describe the provisions of various data cards such as area control AC / A / AO / I, node data B / BD / BM / + and branch data L / LD / LM / T / R / E / RZ. The configuration library is for developers and advanced users to store and collect rules and professional application experience.

ISSN: 0010-8189 
Location aware tracks the user's recent operations and triggers corresponding functions as needed. In addition to menu, toolbar and shortcut keys, location aware can also sense the cursor editing position, mouse position and action as well as the context information of these positions in the editing environment.

Intelligent agent is a program entity with intelligent function and judgment ability. It can activate the output information through location perception, hide it in the editing environment, configure the information in the database according to rules and professional application experience, and actively provide timely and dynamic services for users. The implementation method of intelligent perception is shown in Figure 1. Among many intelligent agents, the best performance includes data card intelligent tracking prompt and error detection, keyword identification and automatic completion, mouse follow-up prompt.

In the simulation data editing, the most frequent operation is to fill in and modify the contents of all kinds of data cards. When filling in the card, you need to select the card type. You should pay attention to the data arrangement, space occupying length, data type and the required blank area. If you are not careful, it will easily lead to data filling errors. By sensing the location of the editing line, the intelligent agent will push the prompt line of the data card to fill in the content nearby. The prompt line changes automatically with the change of editing position, and the data field being edited and the required data field can be displayed in different colors. In the editing window, the edited data field is displayed in color separation according to the length limit, so as to prevent the filling out of bounds.

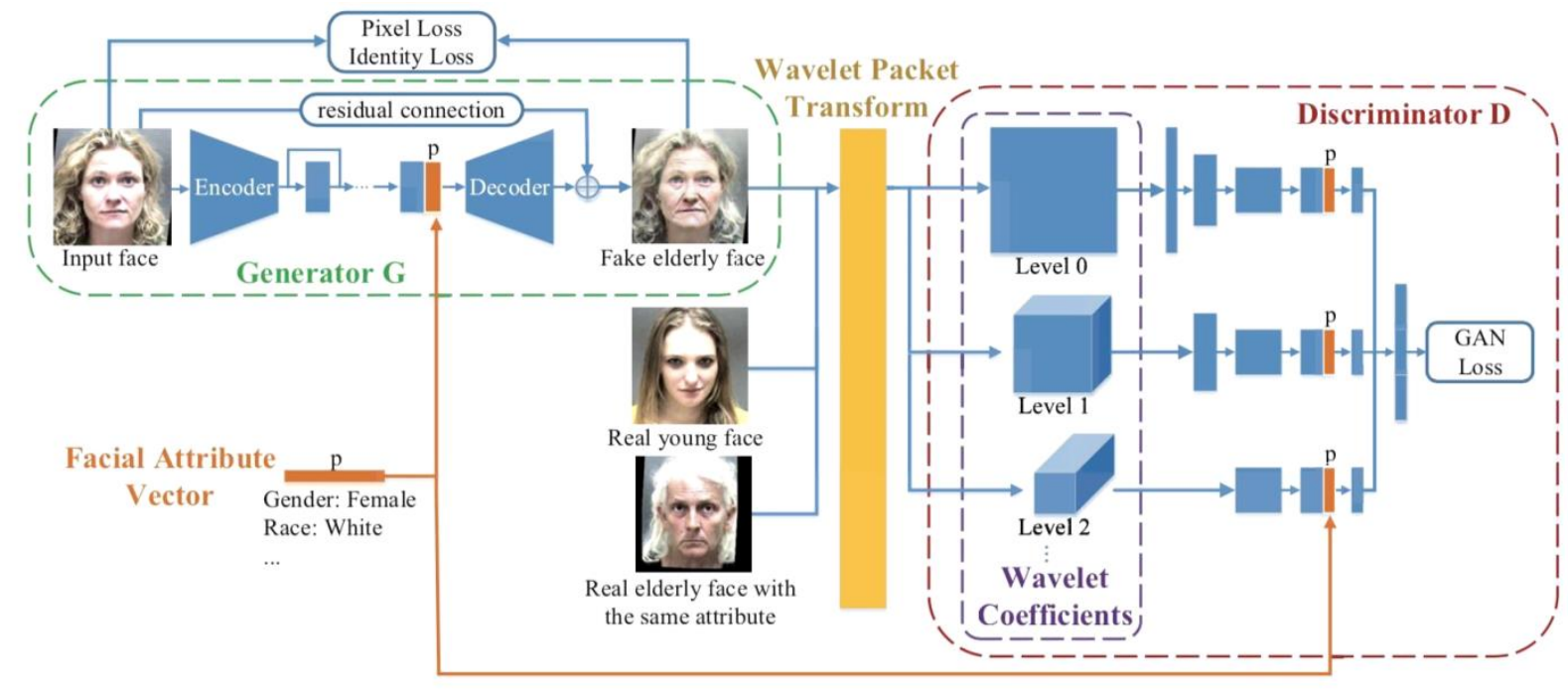

Fig 1: Realization of intelligent perception

When leaving or entering the data line, the intelligent agent will automatically find the filling errors according to the configuration rules, and prompt the error detection results in time. To create a new data card, simply press the hot key to give the data card type code and card type prompt, and fill in the default value of the new line by simple selection. The data card can also be operated in windows. Primary users can fill in the data in the attribute grid window. When filling in, the attribute table will give detailed prompts on the format requirements and value contents of the filled fields.

The existing simulation data editing window is shown in Figure 2 (no intelligent perception screenshot). A completed data card is represented as a segment of characters and data, with no obvious interval between them. For example, the second line of bus data 111511.005 includes three node parameters: voltage, partition and constant active load, so it is difficult for users to quickly distinguish their specific values. After the application of intelligent perception, as long as the mouse position is close to the number string, the number string can be decomposed actively through the intelligent program body, and prompt feedback can be given. For example, when the mouse moves to the middle number 5, the prompt message "row: 12, column: 19-20, partition name: 15" will

ISSN: 0010-8189 
be displayed (see Figure 3). In this way, the meaning of the data can be quickly distinguished, and the timeconsuming operation of moving the edit point back and forth or manual sequence number can be avoided.

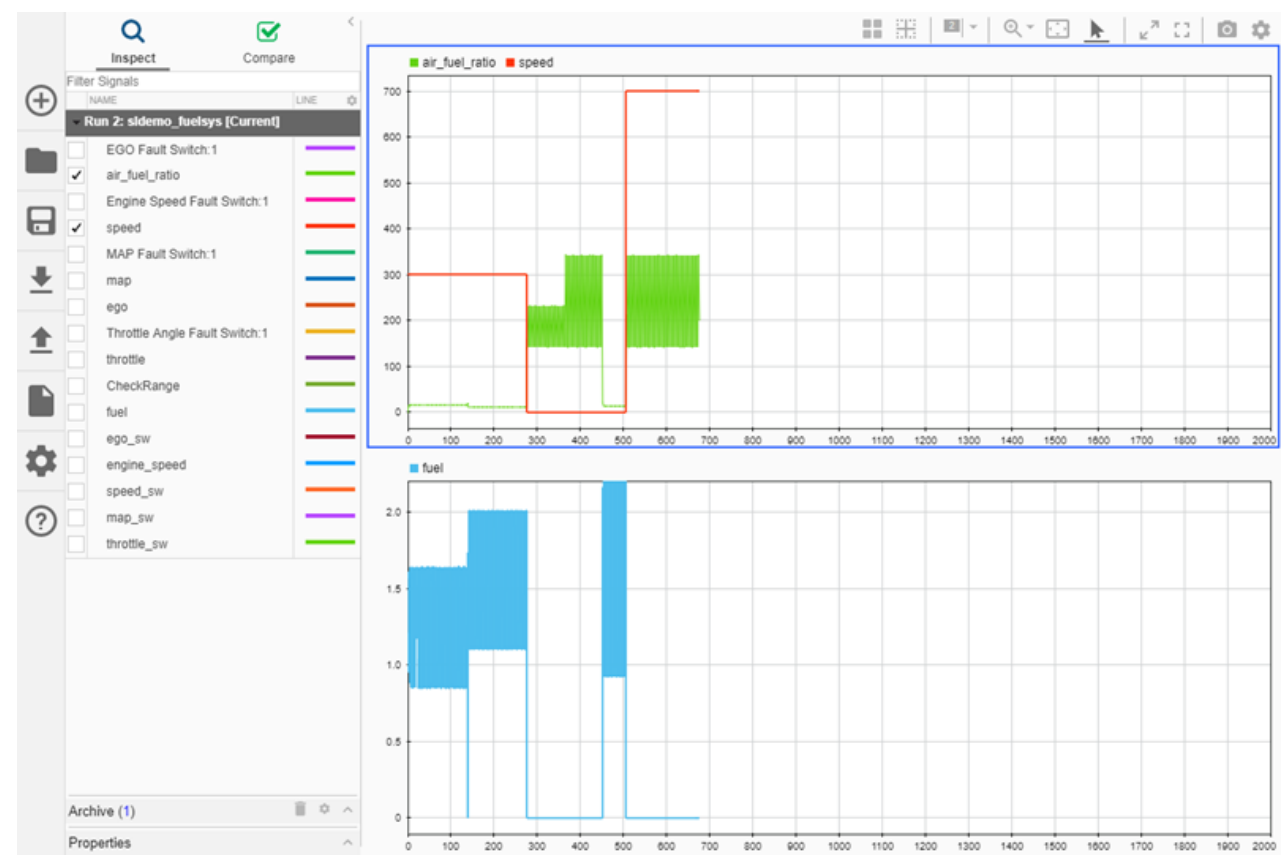

Fig 2: Simulation data window

When editing a control card, IntelliSense provides a set of options for obtaining control keyword references. When filling in or modifying, intelligent perception does not need the data editing software to open another window to perform keyword search, but directly based on the context, automatically finds suitable keywords, and actively promotes the selection list. The filled keywords are displayed in the control card by color separation. When identifying keywords, the intelligent agent uses dynamic lexical analysis technology, which can automatically identify and color with the input or modified content. Keyword intelligent agent sorts and filters the set of all keywords in the background. When the context content changes, it dynamically modifies the list according to the input content, and actively launches an online selection window for users to realize automatic keyword filling. Users can directly insert complete keywords into the control card by simple selection.

\section{Simulation data editing software with intelligent perception}

The application of data editing intelligent perception improves the work efficiency to the greatest extent and saves the time of simulation data construction and modification. Dynamic lexical analysis and automatic completion function lead to the speed of editing doubled. For example, powerflow needs to be typed 9 times. Through automatic completion, you only need to enter $\mathrm{P}$ or PO and select powerflow in the pop-up list window to complete the input easily. The bus name of line parameters, transformer parameters can also be completed by selecting one or more first characters at the input point, which is much faster than copying or direct input.

Automatic prompt of data card type and mouse follow-up prompt, automatic color separation display of data field boundary of edit line, intelligent context prompt based on card type and online error detection of edit line all greatly facilitate data filling and modification. Through the nearby intelligent prompt, it avoids the tedious and time-consuming work of aligning the format back and forth and finding the format error. Users do not need to memorize a wide range of data card regulations, but spend more energy and time to consider the needs and changes of power system simulation.

In the simulation data editing environment, intelligent sensing establishes the location sensing trigger, intelligent judgment and visual output feedback mechanism. The feedback content is recorded in the configuration library

ISSN: 0010-8189 
and maintained by developers and professional users. The configuration library is rich in content, including format definition, data card definition and explanation, precautions, self checking rules. The configuration content itself is placed according to the rules, and the maintainer can expand and revise it according to professional application experience and needs. This design method realizes the decoupling and separation of editing environment, data requirements and simulation calculation. The developer of simulation program can focus on the research and development of simulation model, algorithm and system function, and maintain the requirements of simulation data through configuration library to achieve interaction with users. When users fill in the actual power grid data through the editing environment, they can understand the developer's demand for simulation through intelligent perception. Therefore, the simulation data editing environment based on intelligent perception has strong adaptability and scalability, and is an editing environment for future applications.

The PSD simulation data editing environment developed according to the intelligent sensing implementation method described above is shown in figures 3 and 4 (the power flow data is displayed in the editing window). Simulation data editing environment can open multiple power flow and stable original data files for editing at the same time, and provide intelligent sensing service. At the same time, it is suitable for editing simulation result output files in ASCII code. Figure 3 shows the dynamic prompt effect of the edit window. Figure 4 shows the effect of online error detection and automatic completion. The practice shows that the intelligent data editing environment can greatly reduce the round-trip modification rate, reduce the unconscious errors, and the warning and error location are very convenient, and the usability is greatly enhanced.

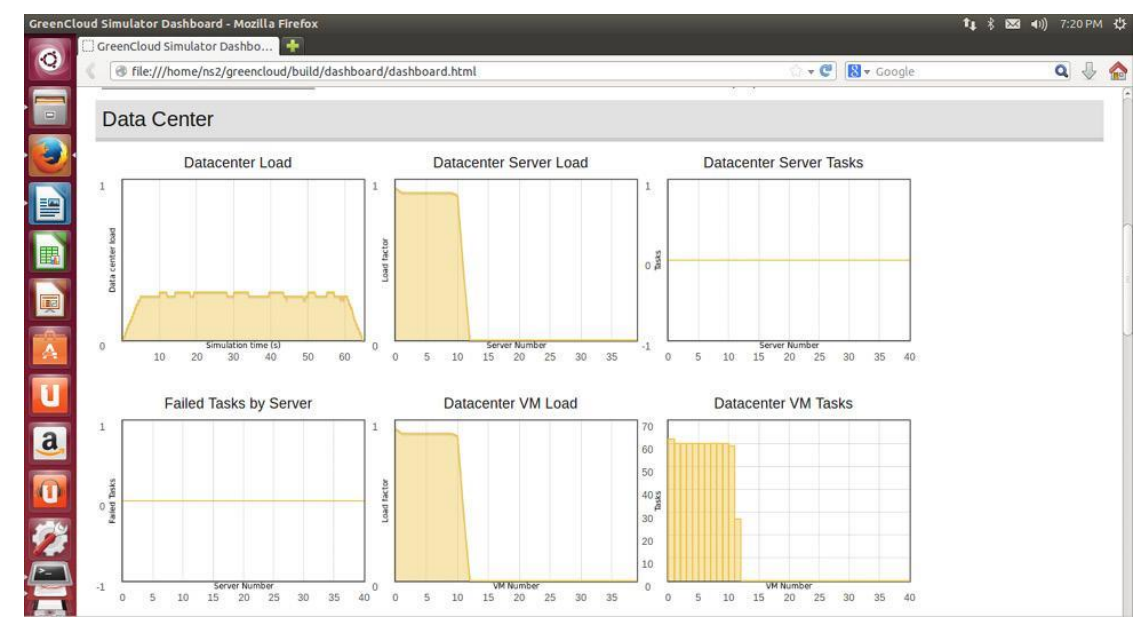

Fig 3: IntelliSense feedback hint window

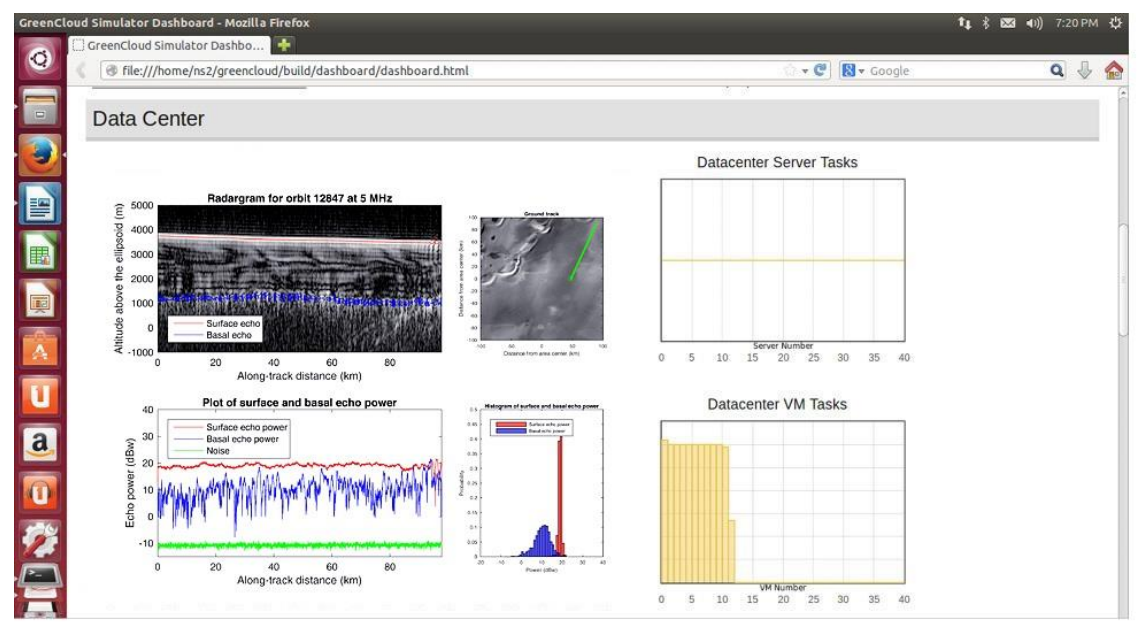

Fig 4: Automatic completion and automatic online error detection prompt window

ISSN: 0010-8189 


\section{Conclusion}

Simulation data editing is the beginning of the simulation process. It is a task that is frequently adjusted in the simulation process and is time-consuming. In order to improve the speed of data creation, reduce errors and enhance ease of use, this paper proposes to use intelligent sensing technology in the simulation data editing environment. The developed PSD data editing software not only retains the characteristics of direct modification of simulation data files, convenient sharing, good independence and data completeness, but also greatly enhances the ease of use and intelligence. The implementation method of intelligent sensing, which integrates location aware, intelligent agent and configuration library, can facilitate developers to upgrade and update simulation kernel and configuration, and has reference value for further research and development of more intelligent and better visual simulation software. The further task is to apply intelligent sensing technology to the statistical analysis of simulation results.

\section{References}

[1] Liu Xiangyang, Wang Dan. Design and Research of Electrical Automation Control System Based on Intelligent Technology. Electronic Design Engineering, 2019, V.27; No.414 (16): $72-75$

[2] Wen Wenhao. Research on Electrical Automation Control System Based on Artificial Intelligence. Information and Computer (theoretical Edition), 2020, V.32; No.446 (04): 152-153

[3] Liu Xiaoyan. Application of Artificial Intelligence Technology in Electrical Automation Control System. Rural Economy and Technology, 2019, 030 (022): 254-255

[4] Guo Yanan, Wang Yanfei. Application of Artificial Intelligence Technology in Electrical Automation Control. Architectural Engineering Technology and Design, 2018, 16: 4184

[5] Huang Qiang. Application of Artificial Intelligence Technology in Electrical Automation Control. Consumer Guide, 2019, 10: 120

[6] Yin Zhangsheng. Research on Electrical Automatic Control Technology Under Artificial Intelligence Technology. Development Orientation of Building Materials, 2019, 017 (009): 363

[7] Wang Li. Application of Artificial Intelligence in Electrical Control System. Full Text Edition: Engineering Technology, 2016 (6): 175-175

[8] Jiang Fkuan, Zhou Rui. Discussion on the Application of Artificial Intelligence Technology in Electrical Automation Control. Dual Use Technologies and Products, 2018, 6: 96

[9] Yao Linhu. Application of Artificial Intelligence in Electrical Automation Control. Intelligent City Application, 2020, 3 (8): 91

[10] Wang Huiqian. Application and Development of Artificial Intelligence Technology in Electrical Control System. Journal of College of Electronic Engineering, 2019, 008 (010): P.137-137 